\title{
CAEP/ACMU 2019 Scientific Abstracts Author Index
}

\begin{tabular}{|c|c|c|c|c|c|}
\hline AUTHOR & ABSTRACT No. & Battaglia F & MP42, P011 & Brehaut J & P083 \\
\hline & & Beach J & LO48, P053 & Brennan E & LO83 \\
\hline $\mathbf{A}$ & & Beamish D & MP46 & Brine $M$ & P055 \\
\hline Abbas K & $\mathrm{P} 131$ & Beane K & LO86 & Brinkhurst J & PL02, LO02 \\
\hline Abdulla S & $\mathrm{LO} 23$ & Beanlands R & MP14 & Brisebois A & MP08, P072 \\
\hline Abu-Laban R & $\mathrm{P} 023$ & Bearss E & P031 & Brison R & PL02 \\
\hline Acton $\mathrm{C}$ & P013 & Beck B & LO08 & Brousseau A & LO13, MP34, P085 \\
\hline Addleman S & P001 & Beckett S & P012, P129 & Brown R & LO73, $\mathrm{P} 060$ \\
\hline Adler S & LO33 & Beirnes N & LO63 & Brown S & $\mathrm{P} 130$ \\
\hline Agarwal G & P085 & Béland E & MP33 & Brubacher J & LO21, MP54 \\
\hline Agarwal S & P002, P032 & Belhumeur V & P043 & Bruneau V & LO57 \\
\hline Aglipay M & P016 & Bell C & LO85 & Brydges $M$ & P085 \\
\hline Aguanno A & P003 & Belle Blagrove L & P015 & Buckley R & P074 \\
\hline Ahluwalia R & LO86 & Benjamin S & MP27, P062 & Bugden $G$ & MP39 \\
\hline Ahmed T & LO81 & Ben-shoshan M & MP55 & Buhariwalla $\mathrm{H}$ & P083 \\
\hline Ainsworth C & MP49, MP52 & Beran T & LO63 & Buisson $\mathrm{C}$ & P084 \\
\hline Akilan K & LO93, MP25 & Berezin L & P091 & Bullard M & LO25, MP15, P119, P120 \\
\hline Alain J & LO69 & Bernard F & LO06, LO09, P028 & Bunker E & P127 \\
\hline Albert $M$ & LO05, LO06, LO09, P028 & Berthelot S & PL04, LO08, LO69, LO70, & Burkoski V & LO88 \\
\hline \multirow[t]{2}{*}{ Ali S } & LO32, LO35, LO36, LO62, & & MP33, MP34, MP48, P056 & Burns K & LO42 \\
\hline & LO63, P102 & Besserer F & P085 & Bursey K & $\mathrm{P} 039, \mathrm{P} 100$ \\
\hline Allain D & MP50 & Bhatt $M$ & P116 & Burwash-Brennan $\mathrm{T}$ & LO42 \\
\hline Almenawer S & MP07 & Bhatt S & LO34 & But & MP37 \\
\hline Alrobaian S & P004 & Bhimani $M$ & P067 & Butter A & LO36 \\
\hline Amat $\mathrm{C}$ & P005 & Biggs C & MP30 & Buxton J & $\mathrm{LO} 20, \mathrm{LO} 21$ \\
\hline Amiro R & P006 & Bignucolo A & $\mathrm{P} 013$ & Byce $S$ & MP43 \\
\hline Amyotte J & MP04 & Bignucolo S & LO04 & Byrne C & LO64 \\
\hline Andruchow J & LO01, LO16, LO24, MP10 & Birnie D & PL02 & & \\
\hline Anjum $\mathrm{O}$ & MP19 & Bismah V & MP04 & & \\
\hline Ansell D & LO04 & Bittira B & LO04 & C & \\
\hline $\operatorname{Arab} M$ & P109 & Black E & MP11 & Callaway Y & LO04 \\
\hline Arbitre C & LO31 & Blain $\mathrm{H}$ & $\mathrm{LO} 25, \mathrm{MP} 15$ & Cameron P & LO08, MP34 \\
\hline Archibald C & $\mathrm{P} 023$ & lais $\mathrm{S}$ & PL04 & Campbell S & LO18, LO19, LO30, P054 \\
\hline Argintaru N & MP18 & laskovits F & LO46 & Campbell SG & MP32, MP37, P015, P071 \\
\hline Arinde $\mathrm{F}$ & $\mathrm{P} 007$ & Blom J & LO28, P128 & Caners K & LO44, LO45 \\
\hline Arora N & P007 & Blondeau B & P066 & Cantor W & MP49, MP52 \\
\hline Ashok D & LO36 & Bobrovitz N & MP02 & Cantor Z & P016 \\
\hline \multirow[t]{2}{*}{ Atkinson P } & LO22, LO42, LO68, & Bodunde $\mathrm{O}$ & LO50, LO51, P014, P106 & Carmichael P & LO11, LO70, MP33, \\
\hline & 05, MP27, P006, P018, P024, & Boilard C & PL04 & & MP35, MP51, P048 \\
\hline $\mathrm{P} 037,1$ & $062, \mathrm{P} 065, \mathrm{P} 069, \mathrm{P} 111, \mathrm{P} 146$ & Bonilla J & MP03 & Carmichael H & LO71 \\
\hline Atoui R & LO04 & Booth R & MP14, LO03 & Carr M & P052 \\
\hline Austin M & P008 & Borgundvaag B & PL02, LO02, LO78, & Carrier $M$ & MP06 \\
\hline \multirow[t]{3}{*}{ Azzam D } & $\mathrm{P} 143$ & & LO81, P031, P091 & Carroll L & LO79 \\
\hline & & Bota $G$ & P096 & Carter A & LO73, P060, P109 \\
\hline & & Bouchard M & PL04 & Casey S & LO92 \\
\hline B & & Boucher V & LO70, MP33, MP35, & Casper $\mathrm{T}$ & LO34 \\
\hline Backus B & LO64 & & P042, P079, P080 & Cassidy C & MP12, P095 \\
\hline Bagshaw S & LO65, MP06 & Boucher E & LO12 & Castellucci L & MP06 \\
\hline Bailey B & LO33 & Boulet J & PL04 & Castonguay V & LO57, MP36, P029, P030 \\
\hline Bakewell F & P009 & Bournelis L & MP28 & Caswell J & LO50 \\
\hline Ballard A & LO33 & Bourque C & LO31 & Cavayas Y & LO05, LO06, LO09, P028 \\
\hline Banerjee A & LO42 & Boutis K & LO59 & Chabot K & P017 \\
\hline Barclay N & MP28, MP31 & Brahmbhatt $\mathrm{S}$ & LO36 & Chacko M & MP18 \\
\hline Baril L & PL04 & Brar R & $\mathrm{LO} 21$ & Chagnon $\mathrm{N}$ & PL01 \\
\hline Bastien D & $\mathrm{P} 010$ & Brasher P & LO20 & Chalker J & P039 \\
\hline
\end{tabular}




\begin{tabular}{|c|c|c|c|c|c|}
\hline Champagne J & MP51, P048 & Cragg A & $\mathrm{LO} 27$ & Dunfield R & P037 \\
\hline \multirow[t]{2}{*}{ Chan T } & LO54, MP41, P061, & Crooks C & P071 & Dunn S & P066 \\
\hline & $\mathrm{P} 125, \mathrm{P} 126, \mathrm{P} 137$ & Crump S & P034 & Dunne C & P038, P039 \\
\hline Chan H & MP54 & Cummings $G$ & P053, LO48 & Dzongkowski E & $\mathrm{LO} 36$ \\
\hline Chandra K & P018 & Curran JA & LO39, MP12, P095, P098 & & \\
\hline Chanyi S & P111 & Curran JH & LO20 & & \\
\hline Chaplin T & LO44, LO45, P112 & Curtis S & LO63 & $\mathbf{E}$ & \\
\hline Charette $M$ & LO71, MP50, P083 & Cyr K & MP50 & Eagles D & LO17, P021 \\
\hline \multirow[t]{2}{*}{ Chartier L } & LO61, LO78, LO86, LO90, & Czosnyka M & MP07 & Eby D & P040 \\
\hline & $\mathrm{LO} 92, \mathrm{P} 078, \mathrm{P} 093, \mathrm{P} 127$ & & & Edgar L & MP16 \\
\hline Chassé P & P064 & & & Eggers $\mathrm{K}$ & P023 \\
\hline Chaudry H & MP14 & D & & Eliyahu L & LO48, P053 \\
\hline \multirow{3}{*}{ Chauny J } & LO05, LO06, LO09, & Dainty K & LO78, MP50, P132, P133 & Elliott C & P041, P139 \\
\hline & MP13, MP36, MP51, & Dakin C & $\mathrm{LO} 44, \mathrm{LO} 45$ & Ellis B & P092 \\
\hline & $\mathrm{P} 028, \mathrm{P} 029, \mathrm{P} 030, \mathrm{P} 048$ & Dale-Tam J & P083 & Elsie S & $\mathrm{LO} 36$ \\
\hline Chen $\mathrm{K}$ & P041, P139 & Daniels N & P071 & Elwi A & MP08, P072 \\
\hline Cheng W & MP07 & Daoust R & LO05, LO06, LO09, LO11, & Emond $M$ & PL01, LO08, LO13, LO70, \\
\hline Chenkin J & LO87, MP21 & & LO70, MP13, MP33, MP36, & & MP33, MP34, P043 \\
\hline Cherry J & P019, P020 & & MP55, P028, P029, P030 & English S & MP07 \\
\hline Cheskes S & LO07 & Dave C & LO65 & Erdelyi S & $\mathrm{LO} 21$ \\
\hline Cheung K & PL01 & Davies D & LO92 & Erdogan $M$ & LO67, P058, P059 \\
\hline \multirow{2}{*}{ Cheung W } & LO17, LO55, LO58, LO84, & Davies-Schinkel C & $\mathrm{P} 130$ & Erker R & LO37 \\
\hline & $\mathrm{P} 026, \mathrm{P} 142, \mathrm{P} 144$ & Davis B & P127 & Estrada-Codecido J & P044 \\
\hline Chhabra S & P021 & Davis $M$ & P086 & Etchells E & LO89 \\
\hline Chiasson D & MP12, P095 & Davis $\mathrm{P}$ & MP54, P115, P121 & Evans C & P008, P104 \\
\hline Chignell M & LO14 & De Leon J & LO86 & Evans J & $\mathrm{LO} 72$ \\
\hline Chin A & $\mathrm{P} 074$ & de Montigny L & LO05, LO06, LO09, P028 & & \\
\hline Chisholm D & $\mathrm{P} 022$ & de Wit K & PL03, LO15, LO23, & & \\
\hline Chorley A & $\mathrm{P} 137$ & & MP40, P088 & $\mathbf{F}$ & \\
\hline Christenson J & $\mathrm{P} 023$ & Denault A & LO05, LO06, LO09, & Falcon C & P089 \\
\hline Church K & $\mathrm{P} 055$ & & MP13, P028 & Fanaeian J & MP30 \\
\hline Cipr & P046 & Deol J & MP30 & Farion $\mathrm{K}$ & LO34 \\
\hline Clar & LO67 & Déry J & MP35 & Fernando S & LO65, MP06, MP07 \\
\hline Clay & PL03 & Deschner E & $\mathrm{P} 031$ & Field S & P110 \\
\hline Clement C & PL02, LO02 & Deshaies J & LO45 & Fielding S & MP30 \\
\hline Cliche J & P017 & Dewhirst S & LO84 & Finamore $\mathrm{S}$ & MP28 \\
\hline Cload B & P108 & Dhanani S & LO66 & Fischer L & MP22, P045, P142 \\
\hline Clouston $\mathrm{R}$ & $\mathrm{P} 006, \mathrm{P} 024$ & Dhani S & $\mathrm{P} 015$ & Fisher A & MP30 \\
\hline Cochrane C & LO21 & Dick A & P129 & Fitzpatrick E & LO39, MP11, P004, \\
\hline Coffey N & LO04 & Diegelmann L & LO22, LO68 & & P019, P020, P036, P134 \\
\hline Coles J & P058, P059 & Dmitriew $M$ & MP43 & Fitzpatrick T & P041 \\
\hline Collings L & LO53 & Dong J & P032 & Fleet $\mathrm{R}$ & P084 \\
\hline Collins D & MP02 & Dong K & LO32, P063 & Fletcher B & MP02 \\
\hline Muret $\mathrm{R}$ & P089 & Dong KJ & P002 & Flynn R & P102 \\
\hline Colun & MP53, P049, P143 & Dong S & P057 & Foote J & P031 \\
\hline Conlon $M$ & LO50 & Dow $\mathrm{N}$ & LO35 & Formosa E & P046 \\
\hline Coriolano K & LO36 & Dowlatshahi D & MP06, MP07 & Forrester S & P047 \\
\hline Corm & LO83 & -Waters M & LO20, LO27 & Forristal C & LO44, LO45 \\
\hline Corn & $\begin{array}{r}\mathrm{P} 024 \\
\mathrm{P} 024\end{array}$ & $S$ & P023 & Fortier C & PL04 \\
\hline Corte & P025, P026 & el A & LO32 & Fortier É & MP51, P048 \\
\hline \multirow{2}{*}{ Cossette S } & LO05, LO06, LO09. & Dreyer J & LO02, P055 & Fortino $\mathrm{N}$ & LO04, P096, P136 \\
\hline & $\begin{array}{r}\text { MP13, P028 }\end{array}$ & Drolet $\mathrm{P}$ & P017 & Foster J & MP47 \\
\hline Costa & LO13, MP34, P085 & Drumm A & LO46, P114 & Fotheringham $M$ & $\mathrm{LO} 45, \mathrm{P} 055$ \\
\hline Costello L & MP18 & Dubois S & MP43 & Fowler M & LO32 \\
\hline Cote A & $\mathrm{P} 027$ & Dudar K & $\mathrm{P} 033$ & Fraccaro L & MP16 \\
\hline \multirow{2}{*}{ Couperthwaite S } & MP08, P075, P076, P077, & Dudek N & LO84 & Franc J & P064 \\
\hline & P147, P148, P149 & Duffy J & P034 & Franczak B & $\mathrm{LO} 47$ \\
\hline \multirow{3}{*}{ Cournoyer A } & LO04 LO05 LO06 LO09 & Duffy $M$ & LO84 & Frank J & 5, LO58, MP22, P001, P142 \\
\hline & LO57 MP13 MP36 MP55, & Duggan S & MP08, P072 & Fraser $\mathrm{J}$ & MP27, P037, P062, \\
\hline & P028, P029, P030 & Dukelow A & LO38, P035, P055 & & P065, P111, P146 \\
\hline $\mathrm{Cox} C$ & MP44 & Duncan J & P036 & Freedman S & $\mathrm{LO} 34, \mathrm{LO} 35$ \\
\hline Crabtree A & $\mathrm{LO} 21$ & Duncan S & P008 & Freeman S & P049 \\
\hline
\end{tabular}




\begin{tabular}{|c|c|c|c|c|c|}
\hline Fremes E & P124 & $\mathbf{H}$ & & $\mathbf{I}$ & \\
\hline \multirow[t]{2}{*}{ French J } & LO22, LO68, MP27, & Ha D & LO45, P057, P081, P117, P118 & Inaba K & MP07 \\
\hline & P006, P037, P062 & Hair H & MP30 & Innes $\mathrm{G}$ & LO01, LO10, LO16, LO24, \\
\hline \multirow[t]{3}{*}{ Froh J } & P121 & Hale S & MP41, P061 & & LO29, LO82, MP10, P068 \\
\hline & & Hall A & P052 & Iseppon $\mathrm{M}$ & MP13 \\
\hline & & Hall AK & LO43, LO44, LO45, & & \\
\hline G & & & LO53, LO56, LO85 & & \\
\hline Gabrielli S & MP55 & Hall J & LO61, P125 & $\mathbf{J}$ & \\
\hline Gagné A & P043 & Halloran B & $\mathrm{LO} 25, \mathrm{MP} 15$ & Jalali H & LO78, P093 \\
\hline Galway L & P114 & Hamad R & P064 & James $M$ & LO01 \\
\hline Gardner K & P050 & Hanel E & $\mathrm{P} 002, \mathrm{P} 032$ & Jarman B & $\mathrm{LO} 42$ \\
\hline Gariepy C & MP51, P048 & Hanlon R & MP27, P062 & Jefkins M & MP43 \\
\hline Garnett M & P033 & Hann J & P063 & Jelic T & MP21 \\
\hline Garrido Clua M & LO30, MP08, P072 & Hanrahan K & MP26 & Jenneson S & MP50 \\
\hline Gatta $M$ & P083 & Harrison M & P109 & Jensen J & $\mathrm{LO} 73, \mathrm{P} 060$ \\
\hline Gaucher N & LO31, P027 & Hartling L & LO63 & Jetty P & LO04 \\
\hline \multirow[t]{2}{*}{ Gaudet L } & LO47, LO48, P051, & Hartmann $\mathrm{R}$ & LO61, MP23, P129 & Jiang A & P067 \\
\hline & $\mathrm{P} 052, \mathrm{P} 053, \mathrm{P} 073$ & Harvey G & MP29 & Jiang A & LO21 \\
\hline \multirow[t]{2}{*}{ Gauri A } & P063, P081, P117, & Hassan A & $\mathrm{P} 111$ & Jiang $M$ & $\mathrm{P} 142$ \\
\hline & $\mathrm{P} 118, \mathrm{P} 119, \mathrm{P} 120$ & Hassani B & P010 & Johnston A & MP17 \\
\hline Ge Y & LO17 & Hau J & LO20, LO27 & Johnston L & P068 \\
\hline Gee A & $\mathrm{P} 144$ & Hayward J & LO29 & Jones A & LO13 \\
\hline Gerein R & LO59 & Hebert G & MP14, P144 & Jones L & P099 \\
\hline Ghalab A & P054 & Hefferon D & LO93, MP25 & Jou H & LO63 \\
\hline Ghosh M & MP30 & Hegg S & LO11, MP51, P043, P048, P094 & Joubert G & LO36 \\
\hline Giffin D & P055 & Heimerl M & LO46, P114 & Jun E & $\mathrm{LO} 32$ \\
\hline Giguère $M$ & MP48, P056 & Hendrikx S & LO62 & Junqueira D & LO18, LO19 \\
\hline Gill S & MP29 & Heneghan C & MP02 & & \\
\hline Giroux M & LO70 & Henneberry $\mathrm{R}$ & LO22, LO68 & & \\
\hline Glanz S & P092 & Hickey Z & P105 & $\mathbf{K}$ & \\
\hline Glicksman R & LO40 & Hicks C & P107 & Kagoma Y & LO15 \\
\hline Godbout J & P083 & Higgins S & P058, P059 & Kanji A & LO22, LO68, P069 \\
\hline Godwin J & LO20, LO21 & Hill N & P077 & Kapend P & $\mathrm{P} 130$ \\
\hline Gofton W & LO84 & Hillier T & P115 & Kapoor A & LO89 \\
\hline Golda N & $\mathrm{P} 074$ & Hirdes J & LO13 & Karol D & P139 \\
\hline Goldstein J & LO41, LO73, P060, P109 & Ho K & P023 & Karram J & LO58 \\
\hline Goodridge D & P140 & Ho V & LO21 & Karreman E & $\mathrm{P} 012$ \\
\hline Gouda J & P149 & Hobden E & $\begin{array}{l}\text { LO21 } \\
\text { LO71 }\end{array}$ & Kasaboski A & P083 \\
\hline Gouin É & LO70, MP33 & Hobden E & $\begin{array}{ll}\text { LU/1 } \\
\text { MP21 }\end{array}$ & Kavsak P & LO01 \\
\hline Gouin S & LO34 & $\begin{array}{l}\text { Hockmann E } \\
\text { Hodoes S }\end{array}$ & $\begin{array}{l}\text { MP21 } \\
\text { MP50 }\end{array}$ & Kearon C & PL03 \\
\hline Gould J & P111 & $\begin{array}{l}\text { Hodges S } \\
\text { Hoffe S }\end{array}$ & $\begin{array}{r}\text { M1P50 } \\
\text { P112 }\end{array}$ & Kelliher B & P070 \\
\hline Grabove $M$ & P022 & $\begin{array}{l}\text { Hoffe S } \\
\text { Hohl C }\end{array}$ & PL02, LO20, LO27 & Kelly N & MP32, P071, P098 \\
\hline \multirow{2}{*}{ Grafstein E } & LO10, LO24, LO82, & Hohl C & $\begin{array}{r}\text { PLOL, LULO, LUL7 } \\
\text { LO45 }\end{array}$ & Kengis L & LO41 \\
\hline & $\begin{array}{r}\text { LU10, LU24, LU82, } \\
\text { MP03, MP10 }\end{array}$ & Holm N & $\begin{array}{l}\mathrm{LO} 45 \\
\mathrm{MP} 30 \mathrm{P} 075 \mathrm{P} 076 \mathrm{P} 077\end{array}$ & Kennedy K & P019 \\
\hline Graham T & LO37, P140 & Holroyd B & $\begin{array}{r}\text { P090, P147, P148 }\end{array}$ & Kester-Greene N & LO43 \\
\hline Graham T & LO35, LO63 & Holt T & LO64 & Kestler A & $\mathrm{LO} 21$ \\
\hline Grainger L & P046 & Homier V & P064 & Keto-Lambert D & $\mathrm{P} 051, \mathrm{P} 073$ \\
\hline Granberg B & MP26 & Hopman W & $\begin{array}{l}1004 \\
\mathrm{LO} 53\end{array}$ & Khadra C & LO33 \\
\hline Gravel G & MP48, P056 & $\begin{array}{l}\text { Hopman W } \\
\text { Horak S }\end{array}$ & $\begin{array}{l}\text { LU53 } \\
\text { MP03 }\end{array}$ & Khalil E & P064 \\
\hline Gravel J & $\begin{array}{r}\text { P031 } 031 \\
\text { P }\end{array}$ & $\begin{array}{l}\text { Horak S } \\
\text { Howlett M }\end{array}$ & LO22, LO68 P065 P111, P146 & Khan S & P074 \\
\hline Gray A & LO90 & $\begin{array}{l}\text { Howlett M } \\
\text { Hoveck P }\end{array}$ & LUL2, LU08, PUO5, P111, P140 & Khazei A & P023 \\
\hline Gray I & $\begin{array}{r}\text { LO90 } \\
\text { P057 }\end{array}$ & $\begin{array}{l}\text { Hoyeck P } \\
\text { Huard B }\end{array}$ & $\begin{array}{l}\text { P138 } \\
\text { PL } 04\end{array}$ & $\operatorname{Kim} \mathrm{A}$ & P104 \\
\hline Gray S & MP09 & $\begin{array}{l}\text { Huard B } \\
\text { Huard R }\end{array}$ & $\begin{array}{l}\text { PLO4 } \\
\text { LO69 }\end{array}$ & Kim J & LO89 \\
\hline Green $M$ & MP45 & Huard K & $\begin{array}{l}\text { LO69 } \\
\text { MP55 }\end{array}$ & Kirk K & LO88 \\
\hline Green R & $\begin{array}{r}\text { N1P45 } \\
\text { P058, P059 }\end{array}$ & Huard $V$ & $\begin{array}{r}\text { MPS5 } \\
\text { L }\end{array}$ & Kirkham A & P063 \\
\hline Greene J & & Huffman J & LO44, LO45 & Kirkland S & LO18, LO19, LO30, MP08, \\
\hline Griesdale D & $\begin{array}{r}\mathrm{O} 73, \mathrm{MP} 49, \text { MP52, Р060 } \\
\text { МР07 }\end{array}$ & Hughes R & P012 & & $\mathrm{P} 051, \mathrm{P} 054, \mathrm{P} 072, \mathrm{P} 073$ \\
\hline $\begin{array}{l}\text { Griesdale D } \\
\text { Grigat D }\end{array}$ & $\begin{array}{r}\text { MP07 } \\
\text { LO25, MP15, P123 }\end{array}$ & Hulme J & MP01, P138 & Kirubarajan A & P074 \\
\hline $\begin{array}{l}\text { Grigat D } \\
\text { Guertin I }\end{array}$ & $\begin{array}{r}\text { LO25, MP15, P123 } \\
\text { PL04 }\end{array}$ & Hung D & MP37 & Kiss A & LO14 \\
\hline Guertin J & PL04 & Hunter S & LO22, LO68 & Kitchen S & I \\
\hline Gupta A & LO04 & Hurley K & LO34, LO39, MP11, P004, & Kitchen S & $\begin{array}{l}\mathrm{LO} 27 \\
\mathrm{MP} 54\end{array}$ \\
\hline \multirow[t]{2}{*}{ Gupta P } & LO36 & & P019, P020, P036, P134 & Koger K & $\begin{array}{r}M P 54 \\
\mathrm{P} 052 \quad \mathrm{P} 075 \quad \mathrm{P} 076\end{array}$ \\
\hline & & Hussain Q & P066 & Krebs L & $\begin{array}{r}\mathrm{P} 052, \mathrm{P} 075, \mathrm{P} 076, \\
\mathrm{P} 077, \mathrm{P} 147, \mathrm{P} 148, \mathrm{P} 149\end{array}$ \\
\hline
\end{tabular}


Kruhlak M LO30, MP08, P052, P054, P072

Krywenky A MP24

Kuehner Z MP43

Kujbid N LO89

Kumar K LO36

Kung J MP03

Kureshi N LO67

Kwok E LO61, LO74, LO79, P021, P078

Kyeremanteng K LO65, MP06, MP07

L

La Vieille S

Lachance A

Lachance-Perreault D

Lafrenière $M$

Lajeunesse $\mathrm{L}$

Lalande E

Lam B

Lam NK

Lam NT

Lamarche $\mathrm{Y}$

Lambert L

Lambert $M$

Lambert S

Lamontagne $M$

Lamprecht $\mathrm{H}$

Landes $M$

Landreville J

Lane D

Lang D

Lang E

LO01, LO04, LO10, LO12, LO16, LO25, MP15, MP30, MP38,

Lanos C P005, P007, P022, P070, P123, P145

Lanoue $M$

Lapointe L

Laroche E

Larocque J

Lavigne $\mathrm{G}$

Lavoie $S$

Law $M$

Le May S

Le Sage N

Leach A

Leach $\mathrm{T}$

LeBouthillier A

Leduc S

Lee A

Lee B

Lee $\mathrm{E}$

Lee J

Lee $M$

Leeper W

Legare J

Legoux C

Lemire $S$

LePage $\mathrm{R}$

Leroux $\mathrm{Y}$

Lesage A

Lessard J

Létourneau M

LO70, MP33

LO50, LO51, P014, P106

LO73, P060

PL04

P029, P030, MP36, MP55

PL04
Levac - Martinho O

Levay D

Lévesque É

Levine A

Lewell M

Lewis D

Leyenaar M

Li J

Lim R

Lin $\mathrm{S}$

Linder B

Lindstrom $\mathrm{R}$

Lingard D

Lipkus $M$

Littlefield S

Liu C

Liu R

Loewen N

Londei-Leduc L

Lonergan A

Loosley J

Loricchio D

Loubani $\mathrm{O}$

Lovas D

Lowthian J

Ludgate $M$

Luhoway J

Lum $\mathrm{H}$

Lund A

Lussier D

Lutchmedial S

Lynch C

Lynch T

Lyon $\mathrm{T}$

Lyster K

M

Ma J

$\mathrm{Ma} \mathrm{K}$

Mabiala Makele P

MacDonald SE

MacDonald SP

MacEachern L

MacInnis $M$

MacIsaac J

MacKinnon C

Macle L

MacMillan K

MacPhee S

Mahajan P

Mahoney K

Mahtani K

Maignan M

Maki C

Mal S

Mallet M
Malo C

Mamdani M

Manaloor R

Maniuk T

Manji S
Manokara $\mathrm{T}$

Marchadier V

Marquis $M$

PL04

LO34

$\mathrm{P} 035$

LO22, LO68, P018

$\mathrm{P} 085$

LO03

MP56

LO52

LO76

$\mathrm{P} 023$

LO72

P086

$\mathrm{P} 033$

LO27

MP56

LO47

LO05, LO06, LO09,

P017, P028

LO45, LO57

$\mathrm{P} 035$

LO76

MP05, MP47

P019

MP34

P096

MP20

LO86

$\mathrm{P} 023$

LO22, LO68

P111

LO76

$\mathrm{P} 132$

P087, P136

P129

LO0 1

LO63

P089

LO35

P088

LO39

MP11

MP16

P023

PL02

MP11

MP11, MP12, P095

LO34

P052

MP02

P089

LO35

P049

PL04, P056

MP51, P043, P048, P094

LO75

LO63

MP46

P007
Marsden J

Martel J

Martin E

Martin L

Maskalyk J

Massaro P

Mastoras G

Matheson K

Mc Ardle B

McCallum J

McCarron J

McColl T

McConnell M

McCracken L

McCredie V

McDonald S

McDonough L

McDuff J

McGowan M

McIsaac D

McIsaac S

McIver K

McKaigney C

McLane $\mathrm{P}$

McLaren J

McLeod B

McLeod L

McLeod S

McParland A

McRae A

Meaney C

Measham J

Medor M

Meiwald A

Mekwan J

Melady D

Melegrito J

Merali Z

Mercier E

Mercuri M

Merlano M

Meshkat N

Meyer J

Michaud S

Middaugh J

Middleton J

Mielniczuk L

Mikhaeil J

Miller M

Milne J

Mitra B

Mittmann N

Moe J

Mok G

Mokhtari A

P086

LO49

LO05, LO06, LO09,

MP13, MP55, P028

$\mathrm{P} 023$

MP29

$\mathrm{P} 023$

MP23, P101

P124

P069

LO44, LO45, MP24, P001

MP11, P004

LO04

LO66

LO81

LO44, LO45

MP42, P011

$\mathrm{P} 007$

MP07

P007

P112

MP28

LO90, MP09, P107, P131

LO65, MP06

LO04, LO49, LO50, P096

LO41

LO85

MP30, P090

MP29

P085

P007

PL03, LO07, LO40, LO78,

LO81, P031, P047,

P091, P092, P133

P141

PL02, LO01, LO02,

LO10, LO16, LO24, MP10

P113

MP50

P142

LO60

LO22, LO68, P111

LO13, MP34, P047, P092

MP26 
Mondal P

Mondoux S

Montpellier O

Montpellier R

Moore L

Morch K

Morin J

Morin M

Morris J

Morrison L

Morrissey L

Mosher L

Mostofa M

Moussienko D

Mowbray F

Mrazik M

Mukarram M

Muldoon K

Murphy N

Murray H

Murray J

Mustafa $\mathrm{H}$

Myre P

Myslik F

\section{$\mathbf{N}$}

Nadeau A

Nasser L

Negreanu D

Nelson M

Nelson R

Nemnom $M$

Nery P

Netherton S

Newbigging J

Newton H

$\mathrm{Ng} B$

Nguyen T

Nichol K

Nolan B

Notebaert É

Nunn J

\section{o}

O'Brien M

O'Brien T

O'Connell K

O'Connor E

Odorizzi S

Ohle R

Olivier P

Olmstead A

Olsen C

Olszynski P

Onakpoya I

P115
P078
P096
PO49
P118
MP48, P056
LO70, MP33
PL01, PL02, LO5, LO66,
LO09, LO57, MP36, MP55,
P017, P028, P029, P030
LO07, MP50
LO91
P004
P065
MP38
LO13, MP34
LO48, P053
MP14, MP46
LO46, P114
P004, P036
PL01
LO75
LO38
MP04
P010

LO70, MP33, MP34, P043

LO61

P094

P047, P092

$\mathrm{P} 036$

LO02, LO03, LO79,

MP14, P083

P083

P066, P115

$\mathrm{P} 105$

LO26

LO21

P003, P049

$\mathrm{P} 045$

P103, P141

LO05, LO06, LO09,

MP13, P028

MP12, P095

LO45

P088

LO34

$\mathrm{P} 034$

LO55

LO04, LO49, LO50, LO51, MP04, P013, P014, P087, P096, P106, P136

PL04

MP30, P081 LO34

P066, P121 MP02
O'Neil B

Orchanian-Cheff A

MP08, P072

$\mathrm{P} 093$

P097

'Rielly C

$\begin{array}{lr}\text { Ospina M } & \text { P075, P076, P077, P147, P148 } \\ \text { Ossip M } & \mathrm{P} 113\end{array}$

Ossip M

Ostrow O

O'Sullivan F

LO78, LO91

Ouellet-Pelletier J

LO20

Ovens H

Owens A

Oyedokun T

Ozog N

LO08, MP48, P056

LO78, LO81, P127

P090

LO37, P108 P140

P098

\section{$\mathbf{P}$}

Pack R

Pageau P

Pan A

P122

Pancino $\mathrm{S}$

P026
P035

Paquet J LO05, LO06, LO09 LO57, MP13,

MP36, MP55, P028, P029, P030

Paquet V

MP51, P048
LO45, LO57, P017
P137
P099

Paradis $M$

Pardhan A

Pardhan K

Parent E

Parent $M$

Park L

Parker R

Parks A

Parpia S

Parsons $M$

Pastore Y

Patel N

Paterson Q

Patrick L

Patrick S

Pavenski K

Pawsey R

Payot A

Peach M

Pearce S

Peddle D

Pelletier M

Penhearow $\mathrm{S}$

Perron R

Perry J

P099

LO33

LO69

MP16

MP47, P134

LO17

PL03

P038, P039, P100

LO31

P105

MP23, P101

MP44

MP24

LO90

P069

$\mathrm{P} 027$

LO22, LO68

$\mathrm{P} 003$

LO72

LO70, MP33

P085

$\mathrm{P} 017$

PL01, PL02, LO02, LO11,

LO65, MP07, P021, P042, P078

Petrie D

LO77

Petrosoniak A LO44, LO45, LO90, MP09

Phalpher P

Pham C

P113

Piette E

LO22, LO68

Pillon S

Pineault A

Piper B

Piraino $\mathrm{P}$

Pishe T

Plint A

Poon J

Poonai N

LO59, P016

MP27, P062

LO32, LO33, LO34, LO35,
Porplycia D

Poss C

Potter B

Powell E

Price L

Price $\mathrm{P}$

Pringle D

Prpic J

Purssell E

Purssell R

Q

Quirion A

P103

\section{$\mathbf{R}$}

Radulovic N

Rajagopal M

Rajajee V

Rajaram A

Ramnanan C

Ramrattan B

Ranger C

Rastogi N

Raymond-Dufresne E

Redley M

Regis A

Reid J

Rich T

Richard C

Richard R

Richardson L

Riggan $M$

Riggs J

Riley J

Risi A

Roback M

Roberts C

Robinson BA

Robinson BE

Robitaille A

Robitaille N

Rocca N

Rochwerg B

Rogers A

Rojas-Luengas $\mathrm{V}$

Rollo D

Roseborough A

Rose-Davis B

Rosgen B

Roskind C

Ross D

Ross M

Rostas B

Rosychuk R

Rotenberg C

Rouleau J

Rouse C

LO36, LO38, LO62, P102

LO61

MP20, P055

LO05, LO06, LO09, P028

LO34

$\mathrm{P} 055$

MP53

MP04

LO20

LO20, LO21

O32, LO35

MP07

P105

MP42, MP16, P011

LO22, LO68

$\mathrm{P} 017$

MP42, P011

LO45

MP40

LO50, LO51, P014, P106

P112

P022

P136

P074

LO22, LO68, MP05

LO28, P128

MP09, P107

$\mathrm{P} 037$

LO21

P116

P108, P129

P146

P109

$\mathrm{P} 017$

LO31

P112

LO65, MP06, MP07

LO34

P091, P133

P111 


\section{Rowe A \\ Rubens F \\ Runham N \\ Rusiecki D \\ Ruske H \\ Russell E \\ Ryan J}

S

Sabhaney V

Sadoway R

Salehi L

Sampsel K

Sandha G

Sandha S

Sandhar G

Sapien R

Savage D

Sayed C

Scales D

Scheirer O

Scheuermeyer $\mathrm{F}$

Schlegelmilch M

Schnadower D

Schonnop R

Schouten A

Schuh S

Schull M

Schweitzer C

Science M

Scott K

Scott SA

Scott SD

Scott T

Seaton B

Sedlakova A

Sedran R

Seely A

Segal E

Segeren $\mathrm{S}$

Sekhon M

Sereda A

Serhan J

Sevcik B

Shah A

Shah S

Shand G

Sharma M

Sheehan C

Sheikh H

Shelton D

Shepherd L

Sheps R

Sherbino J

Sherlock K

Shrier I

Si K

Sibley A

Sibley S

Siddiqi $Z$

Simard R

Simonyan D

\begin{tabular}{|c|c|}
\hline $\mathrm{LO} 21$ & Simpson $M$ \\
\hline LO04 & Sinclair J \\
\hline P149 & Singh V \\
\hline P112 & Sirois $M$ \\
\hline P054, P076, P147 & Sithamparapillai A \\
\hline $\mathrm{LO} 44$ & Sivakumar M \\
\hline MP20 & Sivilotti $M$ \\
\hline & Skeith L \\
\hline & Slack M \\
\hline & Slaunwhite A \\
\hline LO62 & Slaunwhite E \\
\hline MP47 & Smith S \\
\hline P113 & Snyder M \\
\hline LO46, P114 & Socransky S \\
\hline MP15 & Soomro A \\
\hline LO25, MP15 & Sowers N \\
\hline P076, P147 & Speck S \\
\hline LO34 & Spithoff S \\
\hline LO04, LO77 & Spohn J \\
\hline MP42, P011 & Stach J \\
\hline LO52 & Stackhouse S \\
\hline P115 & Stander M \\
\hline LO21, LO24, MP10 & Stauffer B \\
\hline P116 & Steenbeek A \\
\hline $\mathrm{LO} 34$ & Stefan A \\
\hline P117, P118 & Stefanova $\mathrm{V}$ \\
\hline P119, P120 & Stempien J \\
\hline LO34 & Stenstrom R \\
\hline LO10, P127 & Stern E \\
\hline P145 & Stevens $\mathrm{H}$ \\
\hline LO91 & Stewart S \\
\hline MP30 & Stiell A \\
\hline LO81 & Stiell I \\
\hline
\end{tabular}

LO63, P102 LO04

LO78, P133

P121

MP56

LO65

MP50

P122

MP07

P046

P010

$\mathrm{P} 052$

LO62

LO75

MP55

PL01

LO02

MP01, P138

LO87, LO88

P122

LO88

LO55, P125

$\mathrm{P} 022$

LO71

LO89

MP50

LO02

P123

MP18

PL04, LO69
Stiell I

Stoica G

Stoneham E

Stotts G

Strum R

Suleman Z

Sullivan E

Summers J

Suryavanshi T

Sutherland L

Swain J

Swain JM

Swartz R

Swarup V

Syed S

Sykes N

Symons S

\section{$\mathbf{T}$}

Taher A

Talbot J

Taljaard M

Tallon J

Tanguay $\mathrm{R}$

Tanuseputro $\mathrm{P}$

Tarr P

Tavares $W$
Szulewski A
Taylor B

Taylor L

Teefy J

LO11, LO70

P124

LO35, LO63

PL01, PL02, LO76

MP38

LO72

LO21

P020

P100

P040

$\mathrm{P} 013$

$\mathrm{LO} 23$

LO45, MP44

P130

P031

LO62

LO25, MP15

LO82

LO22, LO68

P117

P098

P125

P074

LO37, P129, P140

MP03

LO45

PL01, PL02, LO02, LO17, LO26, LO66, LO71,

MP14, MP45, P083

LO44, LO53, LO56, LO76

Teo V

Termoz Masson N

Thiruganasambandamoorthy V PL02, LO03,

LO79, MP14,

MP46, MP50

Thoma B

Thomas J

Thompson C

Thompson D

Thull-Freedman J

Thurgur L

Tierney $M$

Toarta C

Tolmie A

Tong $\mathrm{T}$

Tran A

Tran AM

Trivedi S

Trottier ED

Tse $\mathrm{S}$

Tung $\mathrm{S}$

Turgeon $\mathrm{J}$

Turgeon R

Turgeon $\mathrm{Z}$

Turner L

LO44, LO45, LO54,

MP23, MP50, P101

LO40

P091, P092

$\mathrm{P} 010$

LO35

LO26, LO55, P025

LO14

LO64, MP14

LO37

LO14

MP40

MP07

LO61, P129

LO31, LO33, LO62

P001

LO76

P079, P080

MP48, P056

LO50, LO51, P014, P106

LO07
LO36

MP44

LO58

LO22, LO68

P101

PL01

P085

MP30

LO37

LO07

P126

P097

MP39

LO73, P060

LO89

LO23

MP19

MP04

LO89

Tutschka M

U

Upadhye S

Urquhart R

P130

Ushko D

P076, P147

V

Vachon K

Vadeboncoeur A

Vaillancourt C

Vaillancourt CA

Vaillancourt S

Valani R

Van Aarsen K

Van de Kleut C

van Hoving $\mathrm{D}$

van Merrienboer J

Vance C

VandenBerg S

Vanderkooi O

LO92, P127

Vandermeer B

Vanosch $M$

Varner C

Vatanpour S

Veldhuyzen van Zanten S

Vella K

Verbeek R

Verdetti A

P111

PL04, MP48, P056

PL02

LO71, LO74, MP50,

MP54, P008, P083 $\mathrm{P} 037$

LO75, P131

P113

LO38, LO60, MP56,

P003, P010, P035,

P055, P086, P132, P143

$\mathrm{P} 132$

LO22, LO68

LO56

LO34

MP29

LO35

LO63

MP28

PL03, LO40, P133

LO01, P123

LO65, MP06

LO34

P085
LO25, MP15

LO56

LO07

P089 


$\begin{array}{lrl}\text { Verma A } & \text { LO89, LO93, MP25 } & \text { White H } \\ \text { Viglino D } & \text { P089 } & \text { Wiercigroch D } \\ \text { Villa-Roel C } & \text { LO18, LO19, LO30, MP08, } & \text { Williams N } \\ & \text { P054, P072, P075, P076, } & \text { Wijdicks E } \\ \text { P077, P147, P148 } & \text { Williamson D } \\ \text { Visser L } & \text { LO18, LO19 } & \text { Wilson B } \\ \text { Voaklander D } & \text { LO48, P053 } & \text { Wilson N } \\ \text { Voyer P } & \text { LO70, MP33 } & \text { Wilson S } \\ & & \text { Wishart I } \\ \text { W } & & \text { Witt L } \\ \text { Wade L } & & \text { Woitowicz J } \\ \text { Wakeling S } & \text { P134 } & \text { Wolfrom B } \\ \text { Walker M } & \text { LO54 } & \text { Wong A } \\ \text { Wallner C } & \text { P112 } & \text { Wong B } \\ \text { Walsh CK } & \text { P135 } & \text { Wong C } \\ \text { Walsh CM } & \text { P031 } & \text { Wong G } \\ \text { Wang A } & \text { LO43 } & \text { Wong M } \\ \text { Wang D } & \text { LO60 } & \text { Wonnacott D } \\ \text { Watson M } & \text { L16005, P022, P070, P145 } & \text { Woo M } \\ \text { Webster Magcalas F } & \text { P136 } & \text { Woo S } \\ \text { Weera S } & \text { LO92 } & \text { Wood T } \\ \text { Wells G } & \text { P130 } & \text { Woodland J } \\ \text { Wells P } & \text { PL01, PL02, MP14, P083 } & \text { Woods R } \\ \text { Welsford M } & \text { MP06 } & \text { Woolfrey K } \\ \text { Welsher A } & \text { MP49, MP52 } & \text { Woolner V } \\ \text { Wharton A } & \text { P137 } & \text { Worster A } \\ \text { Whelan T } & \text { LO49 } & \text { Wu A } \\ & \text { P069 } & \\ & & \end{array}$

$\begin{array}{rlr}\text { P112 } & \text { Wu H } & \text { P063 } \\ \text { MP01, P138 } & \text { Wudwud A } & \text { LO07 } \\ \text { P019, P134 } & & \\ \text { MP07 } & \mathbf{X} & \text { LO35 } \\ \text { MP36 } & \text { Xie J } & \\ \text { LO04 } & & \\ \text { MP39 } & & \text { P094 } \\ \text { P139 } & \text { Y } & \text { LO04 } \\ \text { MP54 } & \text { Yaccarini O } & \text { LO32 } \\ \text { P101, P108, P140 } & \text { Yadav K } & \text { P142 } \\ \text { P002, P032 } & \text { Yan J } & \text { P145 } \\ \text { P105 } & & \text { MP53, P049, P128, P143 } \\ \text { MP18, P141 } & \text { Yaskina M } & \text { PL04 } \\ \text { MP45 } & \text { Yelle Labre K } & \text { LO66 } \\ \text { P097 } & \text { Yeung MEM } & \text { MP22, P001, P144 } \\ \text { MP49, MP52 } & \text { Yeung MYT } & \text { PP22, P001 } \\ \text { P142 } & \text { Yip O } & \text { P004 } \\ \text { P102 } & \text { Yip R } & \text { LO89 } \\ \text { LO42, MP16 } & \text { Yiu S } & \\ \text { LO27 } & \text { Young M } & \\ \text { LO84 } & \text { Yu A } & \text { P037 } \\ \text { MP23, P066 } & & \text { P146 } \\ \text { LO86, LO92 } & \text { Z } & \\ \text { LOnette C } & \end{array}$

